\title{
Fuel Cell Electrode Optimization Through Multi-scale Analytical Microscopy
}

\author{
D.A. Cullen, ${ }^{1}$ B.T. Sneed, ${ }^{2}$ and K.L. More $^{2}$ \\ 1. Materials Science \& Technology Division, ORNL, Oak Ridge, TN USA \\ 2. Center for Nanophase Materials Sciences, ORNL, Oak Ridge, TN USA
}

Polymer electrolyte membrane fuel cells (PEMFCs) are under intense investigation for both transportation and stationary clean energy applications. Controlling the cathode catalyst layer (CCL) structure is a primary area of focus for improving overall PEMFC performance and durability, and ultimately, for reducing cost. The key components of the CCL are the electrocatalyst (typically Pt nanoparticles supported on carbon) for enhancing the oxygen reduction reaction (ORR), ionomer for proton-conduction and electrode mechanical/structural support, and porosity for gas/water transport.[1] These components range in size from single atoms to the micrometer scale, as represented in Fig. 1, and are combined to form the overall CCL structure.

One key to improving electrode performance is the controlled formation of a hierarchical pore structure within the CCL, with secondary micropores $(<2 \mathrm{~nm})$ for transporting oxygen directly to the electrocatalyst surfaces and primary macropores $(>50 \mathrm{~nm})$ for gas and water transport, with mesopores acting as the transport channels between these two length scales. Factors that control this hierarchical structure during CCL processing include the ionomer loading (or the ionomer-to-carbon ratio, I/C), catalyst type and loading, support material, and solvent used during CCL ink preparation. In this work, quantitative analytical scanning transmission electron microscopy (STEM) is employed for highresolution imaging and elemental mapping of the components and their various interfaces within CCLs that have been prepared using different processes. The impact of the support material, electocatalyst (both composition and loading), and the solvent used on CCL morphology, as shown in Fig. 2, will be described in the context of PEMFC performance and durability.

The durability of the CCL components during fuel cell cycling is also of concern. Quantitative electron microscopy methods have previously been used to observe morphological and compositional changes responsible for performance losses during fuel cell conditioning and cycling.[2,3] As an example, electrodes based on novel non-platinum group metal (non-PGM) catalysts, while exhibiting high initial performance, suffer from severe carbon corrosion during fuel cell cycling, leading to rapid thinning of the CCL, as shown in Fig. 3. Complementary imaging and compositional mapping of the CCL constituents at various stages of fuel cell testing will be presented to understand degradation mechanisms, which can be mitigated by modifications to CCL components and membrane electrode assembly fabrication procedures.[4]

\section{References:}

[1] F.A. de Bruijn, V.A.T Dam, and G.J.M. Janssen, Fuel Cells 8 (2008) p. 3.

[2] D. A. Cullen et al., J. Mater. Chem. A 3 (2015) p. 1660.

[3] B. Han et al., Energy Environ. Sci. 8 (2015) p. 258.

[4] Research sponsored by the Fuel Cell Technologies Office, Office of Energy Efficiency and Renewable Energy, U.S. Department of Energy (DOE) and microscopy was conducted at ORNL's Center for Nanophase Materials Sciences (CNMS), which is a U.S. DOE Office of Science User Facility 

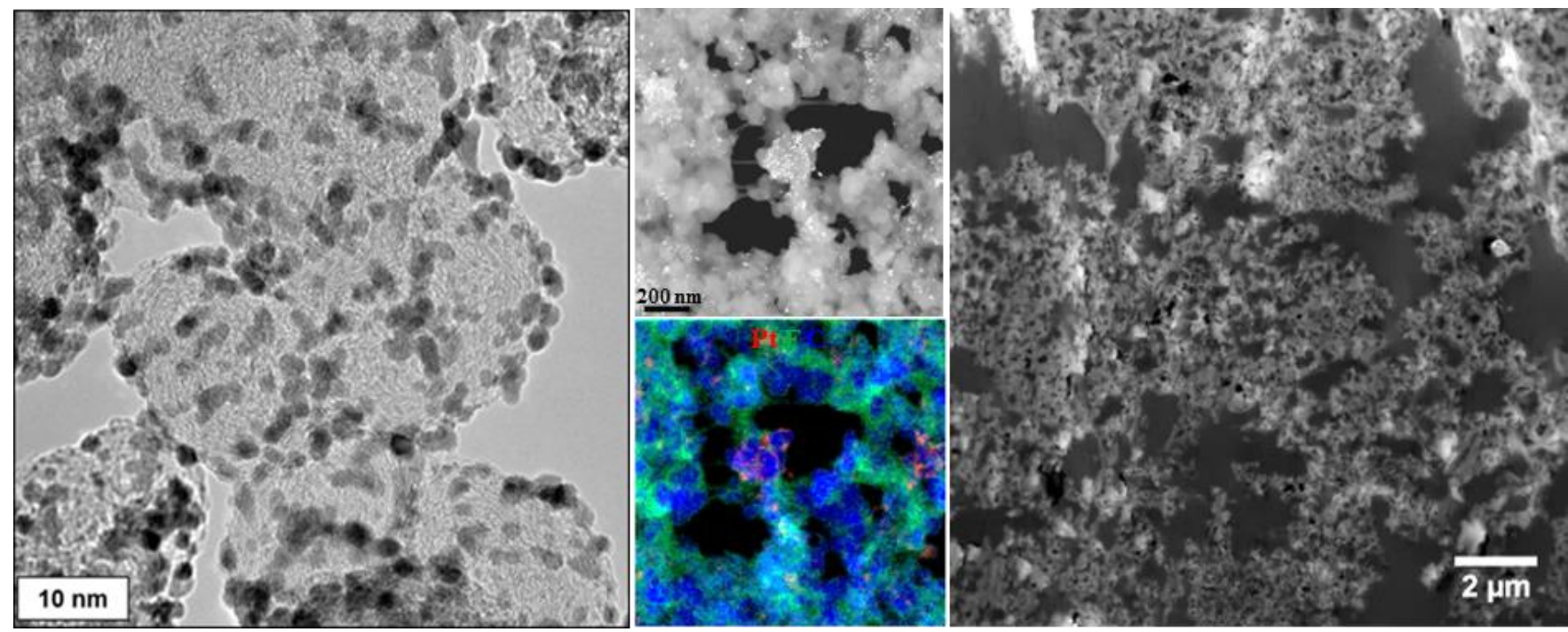

Figure 1. CCLs viewed in cross-section at multiple length scales, from the nanometer scale (left) to micrometer scale (right).

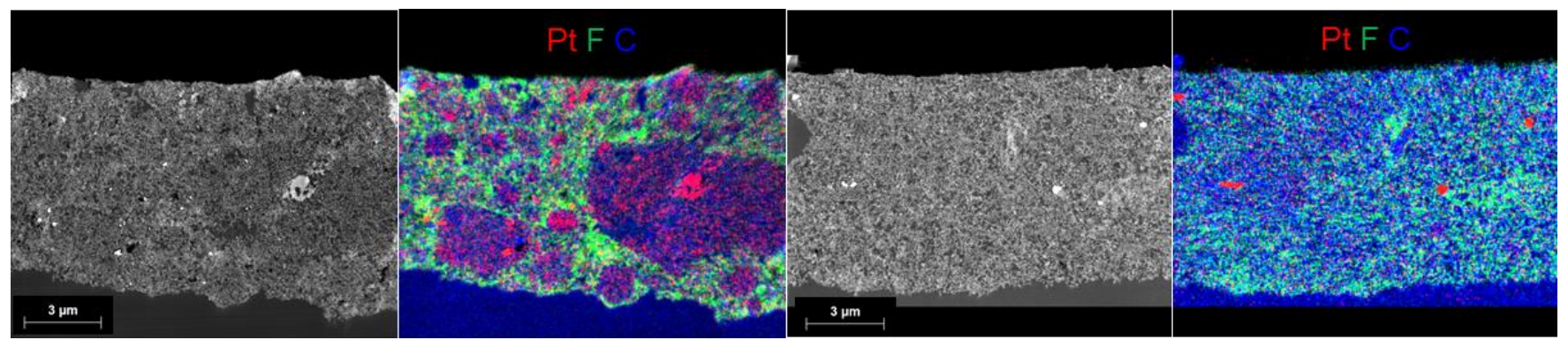

Figure 2. High angle annular dark field (HAADF)-STEM images with energy dispersive X-ray spectroscopy (EDS) spectrum images showing the effect of ionomer loading, catalyst type, and solvent on CCL structure.

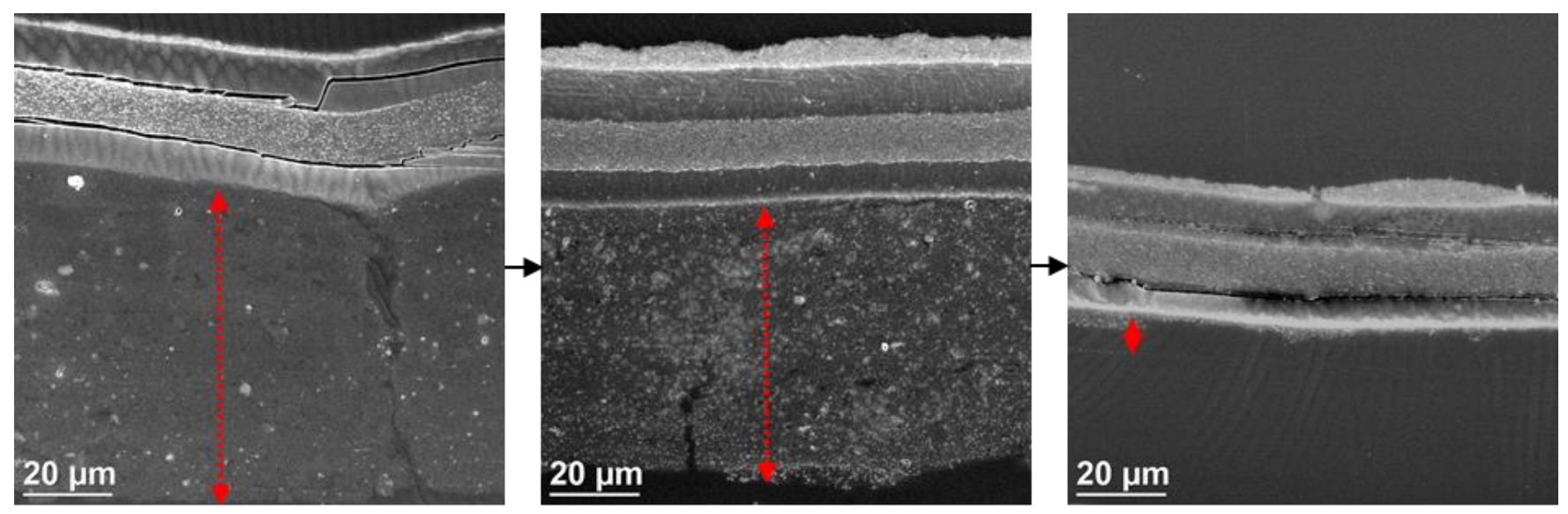

Figure 3. Changes in CCL thickness due to carbon corrosion (CCL thickness shown by red arrows) in a non-PGM CCL at different stages of durability testing. 